\title{
Goniometria dos membros torácicos e pélvicos de ovinos em duas faixas etárias ${ }^{1}$
}

\author{
Renato T. Conceição², Sheila C. Rahal ${ }^{3 *}$, Felipe S. Agostinho ${ }^{4}$, Carlos R. Teixeira ${ }^{3}$, \\ Fábio A.P. Araújo e Frederico O.B. Monteiro ${ }^{5}$
}

\begin{abstract}
Conceição R.T., Rahal S.C., Agostinho F.S., Teixeira C.R., Araújo F.A.P. \& Monteiro F.O.B. 2012. [Goniometric evaluation of both forelimbs and hind limbs in sheep of two different ages.] Goniometria dos membros torácicos e pélvicos de ovinos com duas faixas etárias. Pesquisa Veterinária Brasileira 32(8):812-816. Departamento de Cirurgia e Anestesiologia Veterinária, Faculdade de Medicina Veterinária e Zootecnia, Universidade Estadual Paulista, Campus de Botucatu, Distrito de Rubião Junior s/n, Botucatu, SP 18600970, Brazil. E-mail: sheilacr@fmvz.unesp.br

The purpose of this study was to compare goniometric values for forelimb and hind limbs in clinically healthy Santa Ines sheep with two ages (young and adult). Thirty female Santa Ines sheep were separated into two groups of 15 animals each: Group 1 with 6 to 12-month-old sheep (classified as young), and Group 2 with 3 to 6-year-old sheep (classified as adult). Maximum flexion, maximum extension, and range of motion (ROM) of the shoulder, elbow, carpal, hip, stifle, and tarsal joints, in the right and left limbs were measured with a standard transparent plastic goniometer. The measurements were performed in triplicate by two independent investigators with the sheep in a standing position. There was no statistically significant difference between the right and left sides, between investigators or between groups. In conclusion, in clinically healthy sheep the goniometric values were not influenced by age.
\end{abstract}

INDEX TERMS: Goniometry, angles, sheep, joint, range of motion.

RESUMO.- 0 trabalho teve por objetivo comparar os valores goniométricos das articulações dos membros torácicos e pélvicos em ovinos da raça Santa Inês em duas faixas etárias (jovens e adultos). Foram utilizados 30 ovinos hígidos, fêmeas, divididos em dois grupos: Grupo 1 com 15 animais jovens (idade entre 6 e 12 meses), Grupo 2 com 15 animais adultos (entre 3 e 6 anos). Foram aferidas a máxima flexão, a máxima extensão e calculou-se a amplitude de movimen-

\footnotetext{
${ }^{1}$ Recebido em 9 de março de 2012.

Aceito para publicação em 24 de maio de 2012.

${ }^{2}$ Departamento de Cirurgia e Anestesiologia Veterinária, Faculdade de Medicina Veterinária e Zootecnia (FMVZ), Universidade Estadual Paulista (Unesp), Cx. Postal 560, Distrito de Rubião Junior s/n, Botucatu, SP 18600970, Brasil. Bolsista PIBIC CNPq.

${ }^{3}$ Departamento de Cirurgia e Anestesiologia Veterinária, FMVZ-Unesp, Cx. Postal 560, Distrito de Rubião Junior s/n, Botucatu, SP. *Autor para correspondência: sheilacr@fmvz.unesp.br

${ }^{4}$ Programa de Pós-Graduação em Medicina Veterinária, FMVZ-Unesp, Botucatu, SP.

${ }^{5}$ Instituto de Saúde e Produção Animal, Universidade Federal Rural da Amazônia (UFAM), Av. Presidente Tancredo Neves 2501, Belém, PA 66077530, Brasil.
}

to das articulações, direita e esquerda, dos membros torácicos (ombro, cotovelo e carpo) e pélvicos (coxofemoral, joelho e tarso), com o emprego de um goniômetro universal de plástico. Cada articulação foi aferida em triplicata por dois avaliadores com o animal em estação. Não foram detectadas diferenças estatísticas entre as médias em ambos os lados, entre os avaliadores ou entre os grupos. Foi possível assim concluir que, em ovinos hígidos, os valores goniométricos não foram influenciados pela idade.

TERMOS DE INDEXAÇÃO: Goniometria, ângulos, ovino, articulação, amplitude de movimento.

\section{INTRODUÇÃO}

Em virtude das restrições do uso de animais de estimação em experimentação, especialmente cães e gatos, os ovinos tem se tornado uma opção importante na área de ortopedia e traumatologia (Ehrnberg et al. 1993, Teixeira et al. 2007, Hette et al. 2009, Meller et al. 2009), requerendo assim estudos com relação à função articular. Dentre os métodos objetivos de avaliação das articulações podem ser citados a cinemática, que requer equipamentos mais complexos e analisa 
a geometria do movimento quanto ao deslocamento, à velocidade e à aceleração (Gillete \& Angle 2008), e a goniometria, que apesar de ser uma análise estática, tem por vantagens a simplicidade e o baixo custo (Norkin e White 1997, Jaegger et al. 2002, Hesbach et al. 2007). A fotogrametria computadorizada, que consiste na combinação da fotografia digital e softwares, tem sido comparada a goniometria, porém é ainda pouco utilizada (Sacco et al. 2007, Braz et al. 2008).

A goniometria é um método clínico frequentemente usado por fisioterapeutas e ortopedistas, tanto em Medicina Humana como Veterinária, seja para mensurar o ângulo de uma articulação como sua amplitude de movimento (Gajdosik \& Bohannon 1987, Millis 2004, Hesbach et al. 2007, Knap et al. 2007). Com isso é possível determinar a extensão de uma doença articular, bem como monitorar a progressão de uma lesão e a resposta de um tratamento estipulado (Norkin \& White 1997, Hesbach et al. 2007, Gaiad et al. 2011), tanto no aspecto clínico como experimental. Em Medicina Veterinária a mensuração goniométrica precisa ser efetuada de maneira passiva, ou seja, o movimento articular é efetuado pelo examinador sem a colaboração do paciente, com o mesmo consciente ou não (Mann et al. 1988, Jaegger et al. 2002, Hesbach et al. 2007, Jaegger et al. 2007, Araújo et al. 2009, Gaiad et al. 2011). Em estudos de validação das medidas goniométricas, tanto em cães com em gatos, foi observado que as mensurações obtidas com os animais sedados ou acordados foram válidas e com reprodutibilidade (Jaegger et al. 2002, 2007). Em ovinos da raça Santa Inês foram determinados valores de normalidade com os animais acordados e posicionados em estação (Govoni et al. 2012).

Vários fatores podem interferir na confiabilidade da mensuração goniométrica, como os erros de medida, a variação temporal e a variação biológica em virtude das diferenças entre indivíduos, quanto à idade, sexo, raça e condições clínicas (Gajdosik \& Bohannon 1987, Norkin \& White 1997, Araújo et al. 2009). Com relação à idade, deve-se considerar que o fechamento do disco epifisário cartilagíneo é um determinante do cessar do crescimento ósseo longitudinal de um osso (Moore \& Persaud 2000) e apresenta diferenças entre as espécies animais (Sisson 2008). Particularmente em pequenos ruminantes, a época da fusão do disco epifisário é bastante variável, segundo Sisson (2008). Nos ossos longos dos membros torácicos o período pode variar de 3 meses até 3 anos e meio, de acordo com o osso e disco epifisário cartilagíneo (proximal ou distal), sendo o tempo máximo de 3-4 meses para o úmero, 3 anos e meio para o rádio e para ulna, e 2 anos para o metacárpico III. Nos ossos longos dos membros pélvicos - fêmur, tíbia e fíbula- o período varia de 20 meses a 3 anos e meio.

Baseado nessas premissas, o trabalho teve por objetivo comparar os valores goniométricos das articulações dos membros torácicos e pélvicos em ovinos hígidos da raça Santa Inês em duas faixas etárias (jovens e adultos), para verificar a influência da idade, considerando a padronização do sexo e raça.

\section{MATERIAL E MÉTODOS}

A metodologia adotada no presente trabalho foi aprovada pela Câmara de Ética em Experimentação Animal da Faculdade de Me- dicina Veterinária e Zootecnia da Universidade Estadual Paulista (Unesp) - Botucatu (protocolo $\mathrm{n}^{\circ} .11 / 2011$ - CEUA).

Foram utilizados 30 ovinos hígidos da raça Santa Inês, fêmeas, provenientes de fazendas da região, divididos em dois grupos: Grupo 1 com 15 animais jovens (idade entre 6 e 12 meses), peso corpóreo médio de 25,8 kg (DP = 4,75 kg), e Grupo 2 com 15 animais adultos (entre 3 e 6 anos), peso corpóreo médio 37,6 kg (DP $=2,8 \mathrm{~kg}$ ). Os ovinos foram avaliados por meio de exame clínico geral e ortopédico específico, para descartar quaisquer alterações osteomusculares que pudessem interferir com o experimento.

Um goniômetro universal de plástico transparente foi utilizado para aferir a máxima flexão e a máxima extensão das articulações direita e esquerda, dos membros torácicos (ombro, cotovelo e carpo) e pélvicos (coxofemoral, joelho e tarso), com os animais posicionados em estação, baseando-se nos descritos por Jaegger et al. (2002) e Govoni et al. (2012) (Fig.1). A amplitude do movimento foi calculada pela diferença entre a máxima flexão e máxima extensão. As medidas foram realizadas em triplicata por dois avaliadores.

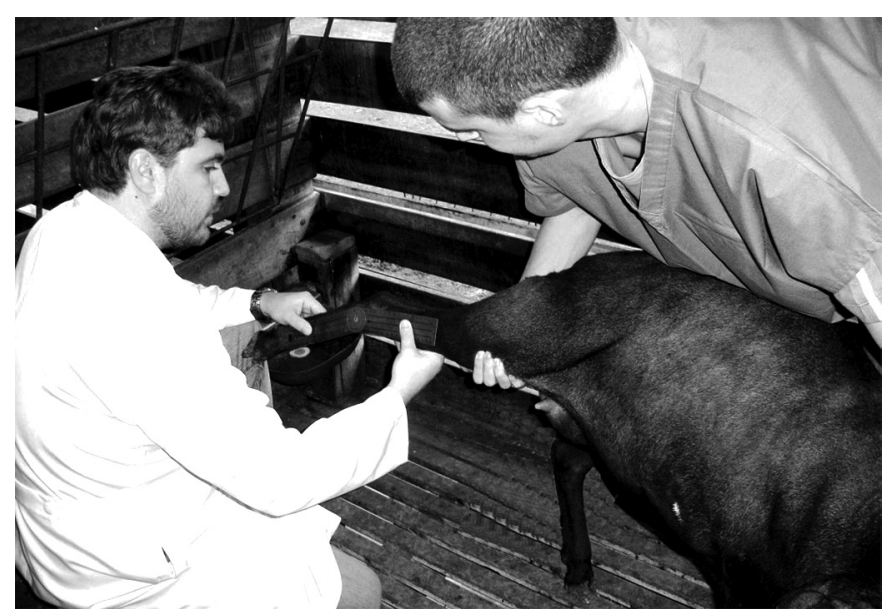

Fig.1. Aferição goniométrica com ovino em posição de estação.

O carpo foi aferido pela colocação do braço móvel do goniômetro ao longo do eixo do osso metacárpico III e IV e o braço fixo ao longo do eixo longitudinal do antebraço, sendo pontos de referência do mesmo o processo estilóide ulnar e o epicôndilo umeral lateral. 0 cotovelo foi aferido pela colocação do braço fixo ao longo do eixo longitudinal do úmero tendo com pontos de referência o epicôndilo umeral lateral e a região de inserção do tendão do infraespinhal no tubérculo maior do úmero, e braço móvel ao longo do eixo longitudinal do antebraço, sendo pontos de referência do mesmo o epicôndilo umeral lateral e o processo estilóide ulnar. Na articulação do ombro o ponto de referência para a centralização do eixo do goniômetro foi à região da inserção do tendão do infraespinhal no tubérculo maior do úmero, sendo que o braço fixo foi posicionado na direção da espinha da escápula, e o braço móvel no eixo longitudinal do úmero tendo como ponto de referência distal o epicôndilo umeral lateral.

$\mathrm{Na}$ articulação do tarso a referência para o centro do goniômetro foi o maléolo lateral, sendo o braço fixo do instrumento posicionado em consonância com o eixo longo da tíbia e o braço móvel com o eixo longo do osso metatársico (III e IV). Para o joelho o ponto de referência para a centralização do goniômetro foi o epicôndilo lateral do fêmur, sendo o braço fixo do instrumento direcionado para o trocânter maior e o braço móvel para o maléolo lateral, em consonância com o eixo longo da tíbia. $\mathrm{Na}$ articulação coxofemoral o ponto de referência para o centro do goniômetro foi o trocânter maior, sendo que o braço fixo do ins- 
Quadro 1. Valores da Máxima flexão (graus) das articulações dos membros torácicos e pélvicos de ovinos hígidos dos Grupos 1 e 2, de acordo com o avaliador e o lado aferido

\begin{tabular}{|c|c|c|c|c|c|c|c|c|c|c|}
\hline \multirow[t]{3}{*}{ Articulação } & \multicolumn{4}{|c|}{ Grupo 1} & \multicolumn{4}{|c|}{ Grupo 2} & \multicolumn{2}{|c|}{ Valor de $\mathrm{p}$} \\
\hline & \multicolumn{2}{|c|}{ Avaliador 1} & \multicolumn{2}{|c|}{ Avaliador 2} & \multicolumn{2}{|c|}{ Avaliador 1} & \multicolumn{2}{|c|}{ Avaliador 2} & \multirow[t]{2}{*}{ Avaliador } & \multirow[t]{2}{*}{ Lado } \\
\hline & $\begin{array}{c}\text { Lado D } \\
\text { (média } \pm D P)\end{array}$ & $\begin{array}{c}\text { Lado } \mathrm{E} \\
\text { (média } \pm \mathrm{DP} \text { ) }\end{array}$ & $\begin{array}{c}\text { Lado D } \\
\text { (média } \pm D P \text { ) }\end{array}$ & $\begin{array}{c}\text { Lado E } \\
\text { (média } \pm D P \text { ) }\end{array}$ & $\begin{array}{c}\text { Lado D } \\
\text { (média } \pm D P \text { ) }\end{array}$ & $\begin{array}{c}\text { Lado E } \\
\text { (média } \pm \mathrm{DP})\end{array}$ & $\begin{array}{c}\text { Lado D } \\
\text { (média } \pm D P \text { ) }\end{array}$ & $\begin{array}{c}\text { Lado } \mathrm{E} \\
\text { (média } \pm \mathrm{DP} \text { ) }\end{array}$ & & \\
\hline Tarso & $36 \pm 3$ & $35 \pm 4$ & $36 \pm 3$ & $35 \pm 4$ & $35 \pm 4$ & $34 \pm 5$ & $35 \pm 3$ & $36 \pm 3$ & 0,31 & 0,09 \\
\hline Joelho & $46 \pm 5$ & $45 \pm 4$ & $44 \pm 2$ & $44 \pm 4$ & $46 \pm 4$ & $45 \pm 3$ & $47 \pm 3$ & $46 \pm 4$ & 0,95 & 0,48 \\
\hline Coxofemoral & $54 \pm 3$ & $53 \pm 3$ & $54 \pm 3$ & $53 \pm 3$ & $54 \pm 4$ & $55 \pm 4$ & $53 \pm 2$ & $53 \pm 2$ & 0,15 & 0,53 \\
\hline Carpo & $20 \pm 1$ & $19 \pm 1$ & $20 \pm 1$ & $20 \pm 1$ & $20 \pm 1$ & $19 \pm 1$ & $20 \pm 1$ & $20 \pm 1$ & 0,24 & 0,39 \\
\hline Cotovelo & $34 \pm 3$ & $34 \pm 4$ & $33 \pm 2$ & $33 \pm 2$ & $34 \pm 3$ & $33 \pm 3$ & $33 \pm 2$ & $33 \pm 2$ & 0,07 & 0,38 \\
\hline Ombro & $88 \pm 2$ & $88 \pm 2$ & $88 \pm 2$ & $88 \pm 3$ & $89 \pm 2$ & $88 \pm 2$ & $88 \pm 2$ & $87 \pm 2$ & 0,15 & 0,54 \\
\hline
\end{tabular}

Quadro 2. Valores da Máxima extensão (graus) das articulações dos membros torácicos e pélvicos de ovinos hígidos dos Grupos 1 e 2, de acordo com o avaliador e o lado aferido

\begin{tabular}{|c|c|c|c|c|c|c|c|c|c|c|}
\hline \multirow[t]{3}{*}{ Articulação } & \multicolumn{4}{|c|}{ Grupo 1} & \multicolumn{4}{|c|}{ Grupo 2} & \multicolumn{2}{|c|}{ Valor de $\mathrm{p}$} \\
\hline & \multicolumn{2}{|c|}{ Avaliador 1} & \multicolumn{2}{|c|}{ Avaliador 2} & \multicolumn{2}{|c|}{ Avaliador 1} & \multicolumn{2}{|c|}{ Avaliador 2} & \multirow[t]{2}{*}{ Avaliador } & \multirow[t]{2}{*}{ Lado } \\
\hline & $\begin{array}{c}\text { Lado D } \\
\text { (média } \pm D P \text { ) }\end{array}$ & $\begin{array}{c}\text { Lado } \mathrm{E} \\
\text { (média } \pm \mathrm{DP})\end{array}$ & $\begin{array}{c}\text { Lado D } \\
\text { (média } \pm D P \text { ) }\end{array}$ & $\begin{array}{c}\text { Lado E } \\
\text { (média } \pm \mathrm{DP})\end{array}$ & $\begin{array}{c}\text { Lado D } \\
\text { (média } \pm D P \text { ) }\end{array}$ & $\begin{array}{c}\text { Lado } \mathrm{E} \\
\text { (média } \pm \mathrm{DP})\end{array}$ & $\begin{array}{c}\text { Lado D } \\
\text { (média } \pm D P \text { ) }\end{array}$ & $\begin{array}{c}\text { Lado } \mathrm{E} \\
\text { (média } \pm \mathrm{DP})\end{array}$ & & \\
\hline Tarso & $161 \pm 5$ & $162 \pm 4$ & $163 \pm 5$ & $164 \pm 5$ & $164 \pm 4$ & $164 \pm 3$ & $163 \pm 3$ & $164 \pm 2$ & 0,62 & 0,12 \\
\hline Joelho & $145 \pm 4$ & $143 \pm 4$ & $145 \pm 5$ & $145 \pm 5$ & $145 \pm 6$ & $145 \pm 6$ & $146 \pm 5$ & $147 \pm 4$ & 0,34 & 0,15 \\
\hline Coxofemoral & $141 \pm 6$ & $140 \pm 8$ & $143 \pm 6$ & $143 \pm 6$ & $143 \pm 6$ & $143 \pm 7$ & $143 \pm 7$ & $144 \pm 7$ & 0,12 & 0,73 \\
\hline Carpo & $171 \pm 2$ & $171 \pm 2$ & $170 \pm 2$ & $171 \pm 2$ & $170 \pm 1$ & $171 \pm 2$ & $170 \pm 1$ & $171 \pm 2$ & 0,55 & 0,16 \\
\hline Cotovelo & $145 \pm 6$ & $144 \pm 5$ & $143 \pm 5$ & $143 \pm 5$ & $144 \pm 9$ & $144 \pm 10$ & $146 \pm 9$ & $146 \pm 8$ & 0,64 & 0,58 \\
\hline Ombro & $145 \pm 8$ & $144 . \pm 7$ & $144 \pm 4$ & $145 \pm 4$ & $142 \pm 5$ & $143 . \pm 6$ & $145 \pm 5$ & $144 \pm 5$ & 0,29 & 0,93 \\
\hline
\end{tabular}

Quadro 3. Valores da Amplitude do movimento (graus) das articulações dos membros torácicos e pélvicos de ovinos hígidos dos Grupos 1 e 2, de acordo com o avaliador e o lado aferido

\begin{tabular}{|c|c|c|c|c|c|c|c|c|c|c|}
\hline \multirow[t]{3}{*}{ Articulação } & \multicolumn{4}{|c|}{ Grupo 1} & \multicolumn{4}{|c|}{ Grupo 2} & \multicolumn{2}{|c|}{ Valor de p } \\
\hline & \multicolumn{2}{|c|}{ Avaliador 1} & \multicolumn{2}{|c|}{ Avaliador 2} & \multicolumn{2}{|c|}{ Avaliador 1} & \multicolumn{2}{|c|}{ Avaliador 2} & \multirow[t]{2}{*}{ Avaliador } & \multirow[t]{2}{*}{ Lado } \\
\hline & $\begin{array}{c}\text { Lado D } \\
\text { (média } \pm D P)\end{array}$ & $\begin{array}{c}\text { Lado E } \\
\text { (média } \pm \mathrm{DP})\end{array}$ & $\begin{array}{c}\text { Lado D } \\
\text { (média } \pm \text { DP) }\end{array}$ & $\begin{array}{c}\text { Lado } \mathrm{E} \\
\text { (média } \pm \mathrm{DP})\end{array}$ & $\begin{array}{c}\text { Lado D } \\
\text { (média } \pm D P)\end{array}$ & $\begin{array}{c}\text { Lado E } \\
\text { (média } \pm \mathrm{DP})\end{array}$ & $\begin{array}{c}\text { Lado D } \\
\text { (média } \pm D P \text { ) }\end{array}$ & $\begin{array}{c}\text { Lado E } \\
\text { (média } \pm \mathrm{DP})\end{array}$ & & \\
\hline Tarso & $126 \pm 6$ & $128 \pm 6$ & $127 \pm 5$ & $128 \pm 5$ & $129 \pm 7$ & $130 \pm 6$ & $128 \pm 4$ & $128 \pm 4$ & 0,63 & 0,08 \\
\hline Joelho & $100 \pm 7$ & $97 \pm 6$ & $101 \pm 5$ & $100 \pm 7$ & $100 \pm 8$ & $100 \pm 6$ & $100 \pm 7$ & $101 \pm 6$ & 0,47 & 0,49 \\
\hline Coxofemoral & $87 \pm 7$ & $87 \pm 9$ & $90 \pm 6$ & $91 \pm 5$ & $89 \pm 7$ & $88 \pm 8$ & $89 \pm 6$ & $91 \pm 6$ & 0,06 & 0,55 \\
\hline Carpo & $151 \pm 3$ & $152 \pm 2$ & $150 \pm 3$ & $151 \pm 3$ & $150 \pm 2$ & $151 \pm 3$ & $151 \pm 2$ & $151 \pm 2$ & 0,33 & 0,09 \\
\hline Cotovelo & $110 \pm 6$ & $111 \pm 6$ & $110 \pm 6$ & $111 \pm 5$ & $110 \pm 9$ & $112 \pm 10$ & $114 \pm 9$ & $114 \pm 8$ & 0,18 & 0,38 \\
\hline Ombro & $57 \pm 8$ & $56 . \pm 8$ & $56 \pm 3$ & $57 \pm 4$ & $53 \pm 6$ & $55 . \pm 6$ & $57 \pm 4$ & $57 \pm 5$ & 0,17 & 0,75 \\
\hline
\end{tabular}

trumento foi posicionado em direção à tuberosidade isquiática e o braço móvel alinhado por uma linha reta que uniu o trocânter maior ao epicôndilo femoral lateral. Os limites máximos da flexão e extensão foram baseados na barreira de resistência à progressão do movimento.

Análises estatísticas descritivas (média \pm desvio padrão) foram calculadas para todos os dados goniométricos. Foi empregado um modelo linear de medidas repetidas com o lado e avaliador como fatores repetidos e grupo como fator entre indivíduos. Diferenças foram consideradas significativas com $p<0,05$. 0 coeficiente de correlação de Cronbach foi usado para determinar a confiabilidade da mensuração conduzida pelo mesmo avaliador (confiabilidade intra-avaliador) e por diferentes avaliadores (confiabilidade inter-avaliador), sendo: $0=$ péssimo; $0-0,20=$ Ruim; $0.21-0,40=$ Regular; $0,41-0,60=$ moderado; $0,61-0,80=$ bom; $0.81-1,00=$ ótimo.

\section{RESULTADOS}

Em virtude do comportamento pouco cooperativo do ovino, as aferições foram sempre realizadas de maneira conjunta pelos dois avaliadores, com um pesquisador realizando a mensuração e o outro mantendo o membro em posição fle- xionada ou estendida. Nas articulações avaliadas não foram observadas diferenças entre as médias em ambos os lados, ou entre os avaliadores. Os valores goniométricos de cada articulação, de acordo com o avaliador, estão descritas nos Quadros 1, 2 e 3.

Não foram detectadas diferenças entre os Grupos 1 e 2 em todas as variáveis estudadas (Quadro 4). Considerando o coeficiente de correlação de Cronbach abaixo de 0,40, mensurações com menor confiabilidade foram as do avaliador 2, em especial no carpo e cotovelo (Quadro 5). A confiabilidade inter-avaliador foi menor que a intra-avaliador e os valores goniométricos foram menos reprodutível nas articulações do joelho e carpo em flexão, considerando o coeficiente de correlação de Cronbach abaixo de 0,20 (Quadro 6).

\section{DISCUSSÃO}

Entre os diversos instrumentos usados para a aferição goniométrica estão o eletrogoniômetro e o goniômetro universal, sendo este último o mais frequentemente empregado em Medicina Veterinária (Norkin \& White 1997, Thomas 
Quadro 4. Valores da Máxima flexão, Máxima extensão e Amplitude do movimento (graus) das articulações dos membros torácicos e pélvicos de ovinos hígidos, de acordo com o Grupo

\begin{tabular}{|c|c|c|c|c|c|c|c|c|c|}
\hline \multirow[t]{2}{*}{ Articulação } & \multicolumn{3}{|c|}{ Flexão } & \multicolumn{3}{|c|}{ Extensão } & \multicolumn{3}{|c|}{ Amplitude do movimento } \\
\hline & G1 & $\mathrm{G} 2$ & $\mathrm{P}$ & G1 & G2 & $\mathrm{P}$ & G1 & G2 & $\mathrm{P}$ \\
\hline Tarso & $35 \pm 4$ & $35 \pm 4$ & 0,75 & $163 \pm 5$ & $164 \pm 3$ & 0,26 & $127 \pm 5$ & $129 \pm 5$ & 0,27 \\
\hline Joelho & $45 \pm 4$ & $46 \pm 4$ & 0,23 & $144 \pm 4$ & $146 \pm 5$ & 0,18 & $100 \pm 6$ & $101 \pm 7$ & 0,76 \\
\hline Coxofemoral & $53 \pm 3$ & $54 \pm 3$ & 0,66 & $142 \pm 7$ & $143 \pm 7$ & 0,50 & $89 \pm 7$ & $89 \pm 7$ & 0,77 \\
\hline Carpo & $20 \pm 1$ & $20 \pm 1$ & 0,26 & $171 \pm 2$ & $171 \pm 2$ & 0,65 & $151 \pm 3$ & $151 \pm 2$ & 0,51 \\
\hline Cotovelo & $33 \pm 3$ & $33 \pm 3$ & 0,58 & $144 \pm 5$ & $145 \pm 9$ & 0,55 & $111 \pm 6$ & $112 \pm 9$ & 0,48 \\
\hline Ombro & $88 \pm 2$ & $88 \pm 2$ & 0,59 & $145 \pm 6$ & $143 \pm 5$ & 0,47 & $57 \pm 6$ & $55 \pm 5$ & 0,44 \\
\hline
\end{tabular}

Quadro 5. Confiabilidade das aferições goniométricas intra-avaliadores em máxima flexão e máxima extensão

\begin{tabular}{lccccc}
\hline \multirow{2}{*}{ Articulação } & \multicolumn{2}{c}{ Máxima flexão } & & \multicolumn{2}{c}{ Máxima extensão } \\
\cline { 2 - 3 } \cline { 5 - 6 } & $\begin{array}{c}\text { Avaliador 1 } \\
\text { Coeficiente }\end{array}$ & $\begin{array}{c}\text { Avaliador 2 } \\
\text { Coeficiente }\end{array}$ & & $\begin{array}{c}\text { Avaliador 1 } \\
\text { Coeficiente }\end{array}$ & $\begin{array}{c}\text { Avaliador 2 } \\
\text { Coeficiente }\end{array}$ \\
\hline Tarso & 0,901 & 0,854 & & 0,919 & 0,881 \\
Joelho & 0,783 & 0,667 & & 0,728 & 0,817 \\
Coxofemoral & 0,606 & 0,586 & & 0,761 & 0,97 \\
Carpo & 0,447 & 0,385 & & 0,592 & 0,216 \\
Cotovelo & 0,304 & 0,205 & & 0,896 & 0,936 \\
Ombro & 0,53 & 0,773 & & 0,92 & 0,836
\end{tabular}

Quadro 6. Confiabilidade das aferições goniométricas inter-avaliadores em máxima flexão e máxima extensão

\begin{tabular}{lcc}
\hline Articulação & $\begin{array}{c}\text { Máxima flexão } \\
\text { Coeficiente }\end{array}$ & $\begin{array}{c}\text { Máxima extensão } \\
\text { Coeficiente }\end{array}$ \\
\hline Tarso & 0,658 & 0,398 \\
Joelho & 0,111 & 0,356 \\
Coxofemoral & 0,431 & 0,415 \\
Carpo & 0,253 & 0,372 \\
Cotovelo & 0,413 & 0,546 \\
Ombro & 0,599 & 0,711
\end{tabular}

et al. 2006, Hesbach et al. 2007). Os goniômetros universais são confeccionados de metal ou de material plástico, como o utilizado no presente estudo, e assemelham-se com um transferidor, constituindo-se basicamente de dois braços, um fixo e um móvel, e um eixo/fulcro (Norkin \& White 1997).

No momento do exame goniométrico, para cada articulação e movimento, o avaliador deve ser capaz de conhecer as posições recomendadas de teste, o posicionamento alternativo, a estabilização necessária, a estrutura e funções articulares, a sensação final normal, os limites ósseos anatômicos, o alinhamento do instrumento (Norkin \& White 1997). No presente estudo o posicionamento do goniômetro nas diferentes articulações seguiu por base o estabelecido em cães por Jaegger et al. (2002), respeitando as diferenças anatômicas, e o determinado em ovinos por Govoni et al. (2012).

Um outro aspecto importante da aferição goniométrica é a confiabilidade da medida intra-avaliador, que representa a habilidade de um único avaliador obter resultados consistentes em mensurações sucessivas, e a inter-avaliador, que representa a concordância entre medidas da mesma articulação por diferentes avaliadores (Rothstein et al. 1983, Gajdosik \& Bohannon 1987, Norkin \& White 1997, Hayes et al. 2001, Araújo et al. 2009). No presente estudo a mensurações com menor confiabilidade foram as do avaliador 2 , em especial no carpo e cotovelo, provavelmente por ser o avaliador menos experiente. Isso reforça a importância do treinamento prévio ao se usar o método em casos clínicos. Por sua vez, a confiabilidade inter-avaliador foi menor que a intra-avaliadores, fato frequentemente citado por outros autores (Gajdosik \& Bohannon 1987, Norkin \& White 1997, Govoni et al. 2012).

Além disso, podem ocorrer variações na confiabilidade da aferição na dependência da articulação analisada, o movimento que desempenha, e as diferenças estruturais e funcionais em termos de ação (Gajdosik \& Bohannon 1987, Norkin \& White 1997, Jaegger et al. 2002). No presente estudo as articulações do joelho e carpo em flexão apresentaram a menor confiabilidade. Esses achados são próximos ao observado por Govoni et al. (2012). Desta forma, essas articulações requerem maior atenção tanto no posicionamento do goniômetro como no momento da manipulação do membro.

Embora os estudos de validade das medidas goniométricas em cães (Jaegger et al. 2002) assim com em gatos (Jaegger et al. 2007) não tenham considerado o sexo como um parâmetro a ser considerado, em pesquisa com pacientes humanos (Soucie et al. 2011), empregando homens e mulheres entre 2 e 69 anos de idade, foi observado que os indivíduos do sexo feminino apresentaram a maior mobilidade articular. Por isso, no presente estudo optou-se por padronizar o sexo, visando à redução dessa possível variável nos resultados das mensurações, assim como Govoni et al. (2012). Na mesma pesquisa referida acima (Soucie et al. 2011), foi notado que a amplitude de movimento para todas as articulações, dos membros superior e inferior, diminuiu com o avanço da idade para ambos os sexos, considerando grupos de 2-8, 9-19, 20-44 e 45-69 anos. Segundo os autores, as diferenças relacionadas à idade podem estar associadas à influência das mudanças fisiológicas do sistema músculo-esquelético decorrentes do envelhecimento, como perda da resiliência da cartilagem, diminuição da força do músculo esquelético, redução da elasticidade dos ligamentos e redistribuição da gordura. Isso diferiu do observado nas ovelhas, considerando um grupo de animais jovens e outro de adulto, já que não houve diferenças estatísticas.

Alguns fatores devem ser considerados, como o tipo de crescimento da ovelha, que apesar de sexualmente madura entre 6 e 9 meses (Jainudeen et al. 2004), apresentam fusão tardia das placas fisárias, com alguns ossos chegando até 3 anos e meio (Sisson 2008). Isso pode promover uma diferença na dinâmica articular, aliado à posição quadrupedal. Ademais o grupo adulto foi constituído de indivíduos com até 6 anos, que podem estar numa faixa etária em que 
as mudanças decorrentes do envelhecimento não se fizeram ainda presentes o suficiente para promover alterações, considerando que a expectativa de vida da ovelha tem sido referida em torno de 10 a 12 anos (Khan et al. 2003).

Foi possível assim concluir que, em ovinos hígidos da raça Santa Inês, os valores goniométricos não foram influenciados pela idade. Contudo, mais estudos seriam necessários com animais senescentes.

Agradecimentos.- À Fundação de Amparo à Pesquisa do Estado de São Paulo (FAPESP) pela bolsa de Iniciação Científica; ao Conselho Nacional de Desenvolvimento Científico e Tecnológico (CNPq) pela bolsa PQ (CNPq); à Coordenação de Aperfeiçoamento de Pessoal de Nível Superior (CAPES) PROCAD Novas Fronteiras 2009.

\section{REFERÊNCIAS}

Araújo F.A.P., Rahal S.C., Machado M.R.F., Teixeira C.R., Lorena S.E.R.S. \& Barbosa L. 2009. Goniometria dos membros pélvicos de pacas (Cuniculus paca) criadas em cativeiro. Pesq. Vet. Bras. 29:1004-1008.

Braz R.G., Goes F.P.C. \& Carvalho G.A. 2008. Confiabilidade e validade de medidas angulares por meio do software para avaliação postural. Fisioter. Mov. 21:117-126.

Ehrnberg A., De Pablos, J., Martinez-Lotti G., Kreicbergs A. \& Nilsson 0. 1993. Comparison of demineralized allogeneic bone matrix grafting (the Urist procedure) and the Ilizarov procedure in large diaphyseal defects in sheep. J. Orthop. Res. 11:438-447.

Gaiad T.P., Silva M.B.,. Silva G.C.A, Caromano F.A., Miglino M.A. \& Ambrósio C.E. 2011. Physical therapy assessment tools to evaluate disease progression and phenotype variability in Golden Retriever muscular dystrophy. Res. Vet. Sci. 91:188-193.

Gajdosik R.L. \& Bohannon R.W. 1987. Clinical measurement of range of motion: Review of goniometry emphasizing reliability and validity. Phys. Ther. 67:1867-1872.

Gillette R.L. \& Angle T.C. 2008. Recent developments in canine locomotor analysis: A review. Vet. J. 178:165-176.

Govoni V.M., Rahal S.C., Agostinho F.S., Conceição R.T., Tsunemi M.H. \& El-Warrak A.O. 2012. Goniometric measurements of the forelimb and hindlimb joints in sheep. Vet. Comp. Orthop. Traumatol. 25: Epub ahead of print.

Hayes K., Walton J.R., Szomor Z.L. \& Murrell G.A.C. 2001. Reliability of five methods for assessing shoulder range of motion. Aust. J. Physiother. 47:289-294.

Hesbach A.L. 2007. Techniques for objective outcome assessment. Clin. Tech. Small Anim. Pract. 22:146-154.

Hette K., Rahal S.C., Volpi R.S., Pereira-Júnior O.C.M., Mamprim M.J. \& Silva V.C. 2009. Radiographic measurement of tibial joint angles in sheep. Vet. Comp. Orthop. Traumatol. 22:204-209.
Jaegger G., Marcelline-Little D.J. \& Levine D. 2002. Reliability of goniometry in Labrador Retrievers. Am. J. Vet. Res. 63:979-986.

Jaegger G., Marcellin-Little D.J., Depuy V., Duncan B. \& Lascelles B.D.X. 2007. Validity of goniometry joint measurements in cats. Am. J. Vet. Res. 68:822-826.

Jainudeen M.R., Wahid H. \& Hafez E.S.E. 2004. Ovinos e caprinos, p.172182. In: Hafez E.S.E. \& Hafez B. (Eds), Reprodução Animal. Manole, São Paulo. 514p.

Khan B.B., Iqbal A. \& Mustafa M.I. 2003. Sheep and Goat production. Department of Livestock Management, University of Agriculture Faisalabad. $419 \mathrm{p}$.

Knap K., Johnson A.L. \& Schulz K. 2007. Fundamentals of physical rehabilitation, p.111-129. In: Fossum T.W. (Ed..), Small Animal Surgery. Mosby Elsevier, St Louis. 1632p.

Mann F.A., Wagner-Mann C. \& Tangner C.H. 1988. Manual goniometric measurements of the canine pelvic limb. J. Am. Anim. Hosp. Assoc. 24:189-194.

Meller R., Haasper C., Westhoff J., Brand J., Knobloch K., Hankemeier S., Hesse E., Krettek C. \& Jagodzinski M. 2009. An animal model to study ACL reconstruction during growth. Technol. Health Care 17:403-410.

Millis D.L. 2004. Assessing and measuring outcomes, p.211-227. In: Millis D.L., Levine D. \& Taylor R.A. (Eds), Canine Rehabilitation and Physical Therapy. W.B. Saunders, St Louis. 544p.

Moore K.L. \& Persaud T.V.N. 2000. Sistema esquelético, p.383-402. In: Ibid. (Eds), Embriologia Clínica. Guanabara Koogan, Rio de Janeiro.

Norkin C.C. \& White D.J. 1997. Medida do Movimento Articular: manual de goniometria. $2^{\mathrm{a}}$ ed. Artes Médicas, Porto Alegre. 260p.

Rothstein J.M., Miller P.J. \& Roettger R.F. 1983. Goniometric reliability in a clinical setting. Elbow and knee measurements. Phys. Ther. 63:16111615.

Sacco I.C.N., Alibert S., Queiroz B.W.C., Pripas D., Kieling I., Kimura A.A., Sellmer A.E., Malvestio R.A. \& Sera M.T. 2007. Reliability of photogrammetry in relation to goniometry for postural lower limb assessment. Rev. Bras. Fisioter. 11:411-17.

Sisson S. 2008. Osteologia, p.693-735. In: Ibid. (Eds), Getty R., Sisson e Grossman, Anatomia dos Animais Domésticos. Guanabara Koogan, Rio de Janeiro.

Soucie J.M., Wang C., Forsyth A., Funk S., Denny M., Roach K.E. \& Boone D. 2011. Range of motion measurements: Reference values and a database for comparison studies. Haemophilia 17:500-507.

Teixeira C.R., Rahal S.C., Volpi R.S., Taga R., Cestari T.M., Granjeiro J.M., Vulcano L.C. \& Correa M.A. 2007. Tibial segmental bone defect treated with bone plate and cage filled with either xenogeneic composite or autologous cortical bone graft. Vet. Comp. Orthop. Traumat. 20:269-276.

Thomas T.M., Marcellin-Little D.J., Roe S.C., Lascelles B.D.X. \& Brosey B.P. 2006. Comparison of measurements obtained by use of an electrogoniometer and a universal plastic goniometer for the assessment of joint motion in dogs. Am. J. Vet. Res. 76:1974-1979. 